K. AZUKAWA AND J. BURBEA

KODAI MATH. J.

7 (1984), 133-152

\title{
HESSIAN QUARTIC FORMS AND THE BERGMAN METRIC
}

\author{
By Kazuo Azukawa and Jacob Burbea
}

\section{Contents}

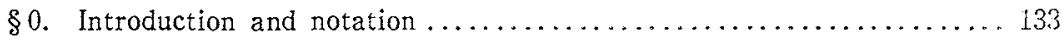

$\S 1$. Hessian quartic form of a hermitian metric $\ldots \ldots \ldots \ldots \ldots \ldots \ldots \ldots .135$

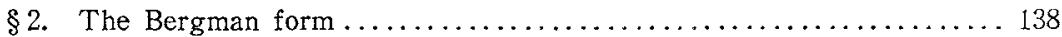

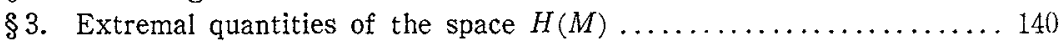

\$4. The biholomorphic invariant $\mu_{0, n} \ldots \ldots \ldots \ldots \ldots \ldots \ldots \ldots \ldots \ldots \ldots$

$\$ 5$. Hessian quartic form of the Bergman metric ................. 147

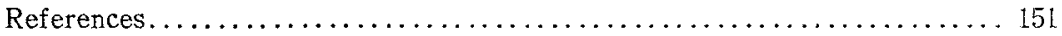

$\S 0$. Introduction and notation. In [7], the "curvature" of the Carathéodory metric on a bounded domain in $C^{m}$ is considered by using the generalized Hessian of this metric; it may be called the Hessian-curvature. Referring to this, we define Hessian quartic forms to an arbitrary hermitian metric. These Hessian quartic forms enable us to provide another proof for the following result of $\mathrm{Wu}$ [14; Lemmas 1 and 4]: The holomorphic sectional curvature coincides with the maximum of the Gaussian curvatures to all local one-dimensional submanifolds that contact at the point in the direction under consideration (Corollary 1.8).

Modifying the construction of the $n$-th order Bergman metric introduced in [6] (also see [5]), we define quantities $\mu_{0, n}(n \in N)$ as follows: We consider a certain linear functional on a specified subspace of square-integrable holomorphic $m$-forms on a $m$-dimensional complex manifold and define the quantity $\mu_{n}$ by the square of the operator norm of this functional (Proposition 3.7). We then set $\mu_{0, n}:=\mu_{n} / \mu_{0}$. The quantity $\mu_{0, n}$ is a $[0,+\infty)$-valued function on the tangent bundle, and is biholomorphic invariant (Theorem 4.2). Especially $\mu_{0,1}$ is the usual Bergman metric, and $2\left(\mu_{0,1}\right)^{2}-\mu_{0,2}$ is the quartic form defining the holomorphic sectional curvature of the Bergman metric (Theorem 4.4).

Let $\lambda_{0, n}^{z}$ be the $n$-th order Bergman metric on a complex manifold, relative to a coordinate $z$, as introduced in [6]. Then the Hessian quartic form of the Bergman metric coincides with $2\left(\mu_{0,1}\right)^{2}-\lambda_{0,2}^{z}$ (Corollary 5.4). In general, $\lambda_{0,2}^{z} \geqq \mu_{0,2}$ with an explicit statement as to when equality holds (Proposition 5.5). Finally, we note that the quantity $\lambda_{0,2}^{z}$ does depend on the coordinate $z$, by examining a concrete example (Corollary 5.8). One should observe, however, that while the quantity $\lambda_{0, n}^{z}$ with $n \geqq 2$ is biholomorphic invariant in the weak sense mentioned

Received March 31, 1983 
in $[5,6]$, it is nevertheless dependent on the coordinate $z$, that is one cannot regard it as a global function on the tangent bundle of the manifold.

Notation. The following notation will be used throughout the paper.

\subsection{Matrices.}

(0.1.1) For a positive integer $n \in \boldsymbol{N}$, we put:

$M(n, C):=$ the set of all $(n, n)$-matrices over $C$.

$G L(n, C):=\{A \in M(n, C)$; det $A \neq 0\}$.

$S(n, C):=\{A \in M(n, C) ; A$ is symmetric $\}$.

$H(n, C):=\{A \in M(n, C) ; A$ is hermitian $\}$.

$P S(n, C):=\{A \in H(n, C) ; A$ is positive semi-definite $\}$.

$P(n, C):=\{A \in H(n, C) ; A$ is positive definite $\}$.

(0.1.2) For $A \in P s(n, C)$, we denote by $A^{1 / 2}$ the square-root of $A$ in $\operatorname{Ps}(n, C)$. If $A \in P(n, C)$ we put $A^{-1 / 2}:=\left(A^{-1}\right)^{1 / 2}$, where $A^{-1}$ is the inverse matrix of $A$ (note that $A^{-1 / 2} \in P(n, C)$ ).

\subsection{Manifolds.}

(0.2.1) The letter " $M$ " will always mean a paracompact connected complex manifold, while the letter " $m$ " designates its complex dimension. The term "coordinate $z$ " stands for a local coordinate system $z=\left(z^{1}, \cdots, z^{m}\right)$ in $M$ with defining domain " $U_{z}$ ". We write $\partial_{a}^{z}:=\partial / \partial z^{a}(a=1, \cdots, m)$, for simplicity.

(0.2.2) For a point $p \in M$, we set:

$T_{p}(M):=$ the holomorphic tangent space at $p$.

$T(M):=$ the holomorphic tangent bundle of $M$.

$\Lambda_{p}^{(s, t)}(M):=$ the space of all $(s, t)$-forms at $p$.

(0.2.3) For a pair of coordinates $z$ and $w$ in $M$ with $U_{z} \cap U_{w} \neq \phi$, we denote by $J_{z}^{w}$ the Jacobian of $w^{\circ} z^{-1}$, i. e. $J_{z}^{w}:=\operatorname{det}\left(\partial_{a}^{z} \cdot w^{b}\right)_{a, b}$.

(0.2.4) For a coordinate $z=\left(z^{1}, \cdots, z^{m}\right)$, we put $d z:=d z^{1} \wedge \cdots \wedge d z^{m}$. The pullback of the euclidian volume element on $C^{m}$ by $z$ is given by $\left(\sqrt{-1^{m}} / 2^{m}\right) d z \wedge d \bar{z}$.

\subsection{Multi-indices.}

Let $m$ be the dimension of $M$ as in (0.2.1).

(0.3.1) Let $M I(n):=\{1, \cdots, m\}^{n}, M I I(n):=\left\{\left(a_{1}, \cdots, a_{n}\right) \in M I(n) ; a_{2} \leqq a_{\imath+1}\right.$ $(\imath=1, \cdots, n-1)\} \quad(n \in N)$, and $M I(0):=M I I(0)=\{\phi\}$. By a multi-index (resp. an increasing multi-index) of length $n$ we mean an element of $M I(n)$ (resp. $M I I(n)$ ).

(0.3.2) For a pair of increasing multi-indices $A=\left(a_{1}, \cdots, a_{n}\right)$ and $B=\left(b_{1}, \cdots\right.$, $\left.b_{n^{\prime}}\right)$, we write $A<B$ if $n<n^{\prime}$ or if $n=n^{\prime}$ implies that $a_{\imath}=b_{i}\left(i<i_{0}\right)$ and $a_{i_{0}}<b_{i_{0}}$ for some $\imath_{n} \in\{1, \cdots, n\}$. 
(0.3.3) For a non-negative integer $n \in \boldsymbol{Z}_{+}$, we denote by $\varphi(n)$ the cardinality of the set $\bigcup_{j=0}^{n} M I I(j)$. Thus $\varphi(n)=\left(\begin{array}{c}m+n \\ n\end{array}\right)$, while the cardinality of $M I I(n)$ is $\varphi(n)-\varphi(n-1)=\left(\begin{array}{c}m+n-1 \\ n\end{array}\right)$ with $\varphi(-1):=0$.

(0.3.4) We denote by $\Phi$ the unique order-preserving bijection from $N$ onto $\bigcup_{n=0}^{\infty} M I I(n)$. Thus, for an increasing multi-index $A$ and for $n \in N$ we have $A \in M I I(n)$ if and only if $\Phi(\varphi(n-1))<A \leqq \Phi(\varphi(n))$.

\subsection{Local differential operators.}

Let $z=\left(z^{1}, \cdots, z^{m}\right)$ be a coordinate in $M$.

(0.4.1) For a constant vector $v=\left(v^{1}, \cdots, v^{i n}\right)$ in $\boldsymbol{C}^{m}$ we put (see $\left.(0.2 .1)\right)$ : $\partial_{v}^{z}:=\sum v^{a} \partial_{a}^{z},\left(\partial_{v}^{z}\right)^{0}:=1^{z},\left(\partial_{v}^{z}\right)^{n}:=\partial_{v}^{z}\left(\partial_{v}^{z}\right)^{n-1}(n=1,2, \cdots)$, where $1^{z}$ stands for the identity operator on functions on $U_{z}$.

(0.4.2) For a multi-index $A=\left(a_{1}, \cdots, a_{n}\right)$ we put: $\partial_{A}^{z}:=\partial_{a_{1}}^{z} \cdots \partial_{a_{n}}^{z}$ (when $n=0$ we have $\partial_{\phi}^{z}=1^{z}$ ).

$\S 1$. Hessian quartic form of a hermitian metric. Let $g$ be an arbitrary hermitian metric on $M$, and let $R$ be the hermitian curvature tensor to the metric in the sense of Kobayashi and Nomizu [12; pp. 155-159] (cf. also [11; pp. 37-39]). For a coordinate $z$ in $M$, we put: $g_{z, a \bar{b}}:=g\left(\partial_{a}^{z}, \partial_{\bar{b}}^{z}\right),\left(g_{z}^{\bar{b} a}\right):=\left(g_{z, a \bar{b}}\right)^{-1}, R_{z, a \bar{b} c \bar{d}}:=$ $g\left(R\left(\partial_{c}^{z}, \bar{\partial}_{d}^{\bar{z}}\right) \bar{\partial}_{b}^{\bar{z}}, \partial_{a}^{z}\right)(a, b, c, d \in\{1, \cdots, m\})$. Thus,

$$
R_{z, a \bar{b} c \vec{d}}=\partial_{c}^{z} \partial_{d}^{z}, g_{z, a \bar{b}}-\sum_{s, t} g_{z}^{i s}\left(\partial_{c}^{z}, g_{z, a \bar{l}}\right)\left(\partial_{d l}^{z}, g_{z, s \bar{b}}\right) .
$$

Definition 1.1. For $p \in M$, we define a quartic form $\operatorname{Sec}(p ; \cdot)$ on $T_{n}(M)$ by

$$
\operatorname{Sec}\left(p ;\left(\partial_{\tilde{v}}^{z}\right)_{p}\right):=-\sum R_{z, a \bar{b} c \bar{d}}(p) v^{a} \bar{v}^{b} v^{c} \bar{v}^{d},
$$

where $z$ is a coordinate around $p$ and $v \in C^{m}$ (see (0.4.1)). Since $\operatorname{Sec}(p ; X)$ $\lg (X, \bar{X})^{2}$ is the holomorphic sectional curvature of $g$ in the direction $X \in T_{p}(M)$ $-\{0\}$, we call $\operatorname{Sec}(p ; \cdot)$ the curvature quartic form of $g$ at $p$.

Remark 1.2. Since $R_{z, a \bar{b} c \bar{d}}$ are components of a tensor, the definition of $\operatorname{Sec}(p ; \cdot)$ does not depend on the coordinate $z$ around $p$.

DEFINITION 1.3. For a coordinate $z$ and $v \in C^{m}-\{0\}$, we set $g_{z, v}:=g\left(\partial_{v}^{z}, \partial_{v}^{z}\right)$ $>0$. For $p \in U J_{z}$ we define a quartic form $\operatorname{Hess}^{z}(p ; \cdot)$ on $T_{p}(M)$ as follows:

$$
\operatorname{Hess}^{2}\left(p ;\left(\partial_{v}^{z}\right\rangle_{p}\right):= \begin{cases}-g_{z, v v}(p) \partial_{v}^{z} \partial_{v}^{z} \cdot \log g_{z, v \bar{v}}(p), & v \neq 0 \\ 0, & v=0 .\end{cases}
$$

Since $\partial_{v}^{z} \partial_{v}^{z}$ is a complex Hessian, we call $\operatorname{Hess}^{2}(p ; \cdot)$ the Hessan quartzc form of $g$, at $p$, relative to $z$. 
LEMMA 1.4. Let $g$ be a hermitian metre on $M, z$ a fixed coordinate around $p$ and $v$ a constant vector in $C^{m}-\{0\}$. We consider the complex line $L:=z(p)+C v$ in the space $C^{m}$ and the connected component $M_{1}$ of $z^{-1}(L)$, containing $p$, which is a one-dimensional complex submanfold in $U_{z}$. We denote by Gauss $(p, v ; \cdot)$ the curvature quartic form, at $p$, of the metric induced from $g$ on $M_{1}$. Then, vnewing $T_{p}\left(M_{1}\right)$ as a subspace of $T_{p}(M)$,

$$
\operatorname{Hess}^{z}\left(p ;\left(\partial_{v}^{z}\right)_{p}\right)=\operatorname{Gauss}\left(p, v ;\left(\partial_{v}^{z}\right)_{p}\right) \text {. }
$$

Proof. The mapping $M_{1} \equiv z^{-1}(z(p)+\xi v) \mapsto \xi \in C$, denoted by $t$, is a coordinate in $M_{1}$ around $p$, while the inclusion mapping $\iota: M_{1} \rightarrow M$ may be represented, under the coordinates $t$ and $z$, as $\xi \mapsto z(p)+\xi v$. The induced metric $t^{*} g$ is given by

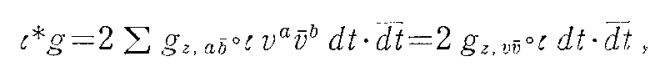

and the hermitian curvature tensor to $\imath^{*} g$ is

$$
{ }^{1} R_{t, 1 \overline{1} \overline{1}}=\hat{\partial}^{t} \partial^{t} \cdot g_{z, v \bar{\nu}} \circ--\left|\hat{\partial}^{t} \cdot g_{z, v \overline{0}} \circ\right|^{2} / g_{z, v \overline{\bar{\nu}} \%} .
$$

Since $\left(\partial_{v}^{z}\right)_{p}=\epsilon_{*}\left(\partial^{t}\right)_{p}=\left(\partial^{t}\right)_{p}$ by the identification of $T_{p}\left(M_{1}\right)$ with $\iota_{*} T_{p}\left(M_{1}\right)$, we have $\left.\operatorname{Gauss}\left(p, v ;\left(\partial_{v}^{z}\right)_{p}\right)=\operatorname{Gauss}\left(p, v ;\left(\partial^{t}\right)_{p}\right)=-{ }^{1} R_{t, 1 \overline{1} 1}(p)=\operatorname{Hess}^{z}\left(p ;\left(\partial_{v}^{z}\right)_{p}\right)\right)$, and the result follows.

Let $(,)_{m}$ (resp. \|\|$_{m}$ ) be the canonical hermitian inner product (resp. the induced norm) on $C^{m}$. Then, for every $p \in U_{z}$ we have $g_{z, v \bar{v}}(p)=v G_{z}(p) v^{*}$ $=\left\|_{v} G_{z}(p)^{1 / 2}\right\|_{m}^{2}$, where $G_{z}:=\left(g_{z}, a \bar{b}\right)$ (see $(0.1 .2)$ ).

PROPOSITION 1.5. Let $g$ be a hermatian metric on $M$, and $z$ be a coordrnate with $G_{z}=\left(g_{z, a \bar{b}}\right)$. Then, for every $(p, v) \in U_{z} \times\left(\boldsymbol{C}^{m}-\{0\}\right)$, we have

$$
\begin{aligned}
& \operatorname{Sec}\left(p ;\left(\partial_{v}^{z}\right)_{p}\right)-\operatorname{Hess}^{2}\left(p ;\left(\partial_{v}^{z}\right)_{p}\right) \\
& \quad=\left(\left\|v A^{1 / 2}\right\|_{m}^{2}\left\|v B A^{-1 / 2}\right\|_{m}^{2}-\left|(v B, v)_{m}\right|^{2}\right) /\left\|v A^{1 / 2}\right\|_{m}^{2}
\end{aligned}
$$

uhere $A:=G_{2}(p)$ and $B:=\hat{\partial}_{v}^{2} \cdot G_{z}(p)$. In partıcular, we have

$$
\operatorname{Hess}^{2}\left(p ;\left(\partial_{v}^{z}\right)_{p}\right) \leqq \operatorname{Sec}\left(p ;\left(\partial_{v}^{z}\right)_{p}\right)
$$

with equality if and only if

$$
v \tilde{o}_{v}^{z} \cdot G_{z}(p)=\xi v G_{z}(p)
$$

for some scalar $\xi \in C$.

Proof. By Definitions 1.1 and 1.3 we have

$$
\begin{aligned}
\operatorname{Sec}\left(p ;\left(\partial_{\eta}^{2}\right)_{\eta}\right)-\operatorname{Hess}^{2}\left(p ;\left(\partial_{v}^{z}\right)_{\eta}\right) & =v B A^{-1} B^{*} v^{*}-\left|v B v^{*}\right|^{2} / v A v^{*} \\
& =\left\|v B A^{-1 / 2}\right\|_{m}^{2}-\left|(v B, v)_{m}\right|^{2} /\left\|v A^{1 / 2}\right\|_{m}^{2} .
\end{aligned}
$$


The last term is zero if and only if $v B A^{-1 / 2}=\xi_{v} A^{1 / 2}$ for some $\xi \in C$. This is equivalent to (1.1) and the proof is complete.

LEMMA 1.6. Let $g$ be a hermitian metrac on $M$, and let $a$ point $p \in M$ and a tangent vector $X \in T_{p}(M)-\{0\}$ be given. Then, there exists a coordinate $z$ around $p$ so that condition (1.1) holds for $v \in C^{m}$ with $X=\left(\partial_{v}^{z}\right)_{p}$.

Proof. We arbitrarily fix a coordinate $w=\left(w^{1}, \cdots, w^{m}\right)$ around $p$ with $w(p)$ $=0$. For every $\left(\xi_{b}^{a}\right) \in G L(m, C)$ and $\left(\xi_{a b}^{c}\right)_{a, b} \in S(m, C)(c=1, \cdots, m)$ (see $(0.1 .1)$ ), the equations

$$
w^{c}=\sum_{a} \xi_{a}^{c} z^{a}+\sum_{a, b} \xi_{a b}^{c} z^{a} z^{b} \quad(c=1, \cdots, m)
$$

define a new coordinate $z=\left(z^{1}, \cdots, z^{m}\right)$ around $p$ with $z(p)=0$ by the inverse mapping theorem. We shall select the numbers $\xi_{a}^{c}, \xi_{a b}^{c}$ so that $z$ satisfies (1.1) for $v \in C^{m}$ with $X=\left(\partial_{v}^{z}\right)_{p}$.

First, we can find a matrix $\left(\xi_{a}^{c}\right)$ so that

$$
v^{a}=0(a=2, \cdots, m), \quad G_{z}(p)=1_{m},
$$

where $G_{z}:=\left(g_{z_{a} a \bar{b}}\right)$ and $1_{m}$ is the identity matrix. Indeed, we set $X_{1}$ : $=X / g(X, \bar{X})^{1 / 2}$ and select $X_{2}, \cdots, X_{m} \in T_{p}(M)$ so that $g\left(X_{a}, \bar{X}_{b}\right)=\delta_{a b}$. If we write $\sum_{c} \xi_{a}^{c}\left(\partial_{c}^{w}\right)_{p}:=X_{a}$, then $\left(\xi_{a}^{c}\right)$ is the desired matrix.

By virtue of (1.3), condition (1.1) is equivalent to

$$
\partial_{1}^{z} \cdot g_{z, 1 \bar{a}}(p)=0 \quad(d=2, \cdots, m) .
$$

Making use of (1.2), condition (1.4) can be rewritten as

$$
\Sigma_{a, b} g_{w, a \bar{b}}(p) \bar{\xi}_{d}^{b} \xi_{11}^{a}=-\frac{1}{2} \Sigma_{a, b, c} \partial_{c}^{w} \cdot g_{w, a \bar{b}}(p) \xi_{1}^{c} \xi_{1}^{a} \bar{\xi}_{d}^{b} \quad(d=2, \cdots, m) .
$$

Since $G_{w}(p)\left(\bar{\xi}_{b}^{a}\right) \in G L(m, C)$, equations (1.5) with unknowns $\xi_{11}^{a}(a=1, \cdots, m)$ possess a solution. This concludes the proof.

Combining the last lemma with Proposition 1.5, we obtain the following assertion :

Proposition 1.7. For $X \in T_{p}(M), \operatorname{Sec}(p ; X)$ conncides with $\max \left\{\operatorname{Hess}^{z}(p ; X)\right.$; $z$ is a coordinate around $p$.

By virtue of Lemma 1.4, this proposition yields the following result which was alluded to in the introduction of this paper.

Corollary 1.8. (Wu [14; Lemmas 1 and 4]). For a tangent vector $X \in T_{p}(M)-\{0\}$, the holomorphic sectional curvature $\operatorname{Sec}(p ; X) / g(X, \bar{X})^{2}$ to a hermitian metric $g$ on $M$ coincides with $\max \left\{G C_{S}(p)\right.$; $S$ is a local one-dimensional submanifold such that $S \ni p$ and $\left.\iota_{S *} T_{p}(S)=C X\right\}$, where $\iota_{S}$ is the inclusion mappings 
of $S$ into $M$, and $G C_{S}(p)$ is the Gaussian curvature at $p$ to the induced metric $\iota_{S}^{*} g$.

Remark 1.9. In [7], a generalized definition of the "Hessian curvature" $\operatorname{Hess}^{z}(p: X) / g(X, \bar{X})^{2}$ is used for the square of the Carathéodory metric on a bounded domain in $C^{m}$.

§2. The Bergman form. We recall the notion of the Bergman form of $M$. For this we follow the description given in $[5,6]$. The set of all holomorphic $m$-forms $\alpha$ on $M$ satisfying $\|\alpha\|^{2}:=\left(\sqrt{-1^{m}} / 2^{m}\right) \int_{M} \alpha \wedge \bar{\alpha}<+\infty$ is denoted by $H(M)$. The space $H(M)$ becomes a pre-Hilbert space over $C$ with an inner product (, ) inherited from the norm \|\| .

Definition 2.1. Let $\alpha$ be a $(m, 0)$-form on $M$, and let $z$ be a coordinate in $M$. We denote by $\alpha_{z}$ the function on $U_{z}$ such that $\left.\alpha\right|_{U_{z}}=\alpha_{z} d z$ (see (0.2.4)).

Applying the Cauchy integral formula to $\alpha_{z}, \alpha \in H(M)$, we find that $H(M)$ is in fact a separable Hilbert space, and, moreover, for a coordinate $z$ around a point $p \in M$ and for a holomorphic differential operator $D^{z}$ on $U_{z}$, the linear functional $H(M) \ni \alpha \mapsto D^{z} . \alpha_{z}(p) \in C$ is bounded (see also Kobayashi [10] and Lichnerowicz [13]). By the Riesz-representation theorem there exists a unique element $\gamma\left(D^{z}, p\right) \in H(M)$ such that

$$
D^{z} \cdot \alpha_{z}(p)=\left(\alpha, \gamma\left(D^{z}, p\right)\right), \quad \alpha \in H(M) .
$$

Especially, when $D^{z}=1^{2}$ (see $(0.4 .1)$ ), we set

$$
\kappa_{z, p}:=\gamma\left(1^{z}, p\right) \text {. }
$$

For another coordinate $w$ around $p$ we have

$$
\kappa_{z, p}=\overline{J_{z}^{w}(p)} \kappa_{w, p},
$$

since $\alpha_{z}=J_{z}^{w} \alpha_{w}$ on $U_{z} \cap U_{w}$ for every $\alpha \in H(M)$ (see $(0.2 .3)$ ).

LEMMA 2.2. Let $\gamma=\gamma\left(D^{z}, p\right)\left(\right.$ resp. $\left.\kappa_{z, p}\right)$ be as in (2.1) (resp. (2.2)). Then, $D^{z} \cdot\left(\kappa_{z, p}\right)_{z}(p)=\overline{\gamma_{2}}(\bar{p})$. follows.

Proof. By definition $D^{2} \cdot\left(\kappa_{z, y}\right)_{z}(p)=\left(\kappa_{z, p}, \gamma\right)=\overline{\left(\gamma, \kappa_{z, p}\right)}=\overline{\gamma_{z}(p)}$, and the result

Let $\bar{M}$ be the conjugate complex manifold of $M$, and denote by $M \ni p \mapsto \bar{p} \in \bar{M}$ the conjugation. For a coordinate $z$ in $M$, we denote by $\bar{z}$ the conjugate coordinate of $z$ with defining domain $\overline{U_{z}}$, i. e. $\bar{z}(\bar{p}):=\overline{z(p)}, p \in U_{z}$.

Definition 2.3. For $p, q \in M$ we set $K(q, \bar{p}):=\kappa_{z, p}(q) \wedge d \bar{z}_{\bar{p}}$, where $z$ is a 
coordinate around $p$. By property (2.3) the quantity $K$ is a well-defined $(2 m, 0)$ form on the product manifold $M \times \bar{M}$ of dimension $2 \mathrm{~m}$, and is called the Bergman form of $M$ (cf. $[5,6]$ ).

Applying Definition 2.1 for the manifold $M \times \bar{M}$, we find that $\left.K\right|_{U_{w}: \bar{U}_{2}}=$ $K_{w \times \bar{z}} d w \wedge d \bar{z}$. On the other hand, by Definition 2.3

$$
K_{w \times \bar{z}}(\cdot, \bar{p})=\left(\kappa_{z, p}\right)_{w}
$$

on $U_{w}$, for every $p \in U_{z}$. It follows from Lemma 2.2 that

$$
K_{w \times \bar{z}}(q, \bar{p})=\overline{K_{2 \times \bar{w}}}(\bar{p}, \bar{q}), \quad(p, q) \in U_{z} \times U_{w} .
$$

By virtue of (2.4) and (2.5), the function $K_{w \times \dot{z}}$ is holomorphic on $U_{w} \times U_{z}$ by Hartogs' theorem of holomorphy. Thus, the Bergman form is a holomorphic $2 m$-form on $M \times \bar{M}$.

DEFINITION 2.4. Let $D$ be a holomorphic differential operator on a coordinate neighborhood $U_{z}$, and let $\omega=\sum_{A \in F} \omega_{A} d z^{A}$ be a holomorphic differential form on $U_{z}$, where $F$ is a finite subset of $\bigcup_{n=0}^{m} M I I(n)$ (see (0.3.1)). Let $d z^{A}:=$ $d z^{a_{1}} \wedge \cdots \wedge d z^{a_{n}}$ for $A=\left(a_{1}, \cdots, a_{n}\right) \in F$. We denote by $D . \omega$ the action of $D$ on $\omega$ coefficient-wise, i.e. $D . \omega:=\Sigma_{A \in F}\left(D . \omega_{A}\right) d z^{A}$. Viewing $\bar{D}$ as a holomorphic differential operator on $M \times \bar{U}_{z}$, we have $D . K(q, \bar{p})=\bar{D} \cdot K_{w \times \bar{z}}(q, \bar{p}) d w_{q} \wedge d \bar{z}_{\bar{p}}$, $(q, \bar{p}) \in U_{w} \times \bar{U}_{\bar{z}}^{-}$. We denote by $\bar{D} . K(\cdot, \bar{q}) / d \bar{z}_{\bar{p}}$ the well-defined holomorphic $m$ form $\beta$ on $M$ such that $\left.\beta\right|_{U_{w}}=\bar{D} . K_{w \times \bar{z}}(\cdot, \bar{p}) d w$ for every coordinate $w$, i.e. $\bar{D} \cdot K(\cdot, \bar{p})=\left(\bar{D} \cdot K(\cdot, \bar{p}) / d \bar{z}_{\bar{p}}\right) \wedge d \bar{z}_{\bar{p}}$.

Proposition 2.5. ([5; Lemma 1], [6; Lemma 1]). Let $D^{z}\left(\right.$ resp. E $\left.{ }^{w}\right)$ be a holomorphic differential operator on a coordinate neighborhood $U_{z}\left(\right.$ resp. $\left.U_{w}\right)$ of $p$ (resp. q). Let $\gamma\left(D^{z}, p\right)$ and $\gamma\left(E^{w}, q\right)$ be as in (2.1). Then:

(i) $\overline{D^{z}} \cdot K(\cdot, \bar{p}) / d \bar{z}_{\bar{p}}=\gamma\left(D^{z}, p\right) \in H(M)$;

(ii) $\left(\gamma\left(D^{z}, p\right), \gamma\left(E^{w}, q\right)\right)=E^{w} \overline{D_{z}} \cdot K_{w \times \bar{z}}(q, \bar{p})$.

Proof. (i) Let $x$ be a coordinate, and let $D:=D^{z}$. Using Lemma 2.2 . (2.4) and (2.5) we have for every $r \in U_{x}$,

$$
\begin{aligned}
r(D, p)_{x}(r) & =D \cdot\left(\bar{\kappa}_{x, r}\right)_{z}(\bar{p}) \\
& =\bar{D} \cdot \bar{K}_{z \times \bar{x}}(p, \bar{r}) \\
& =\bar{D} \cdot \overline{K_{z \times \bar{x}}}(p, \bar{r}) \\
& =\bar{D} \cdot K_{x \times \bar{z}}(r, \bar{p}) .
\end{aligned}
$$

Therefore, $\left.\gamma(D, p)\right|_{U_{x}}=\bar{D} . K_{x \times i}(\cdot, \bar{p}) d x$, as desired.

(ii) By definition and part (i), we have 


$$
\begin{aligned}
\left(\gamma\left(D^{z}, p\right), \gamma\left(E^{w}, q\right)\right) & =E^{w} \cdot \gamma\left(D^{z}, p\right)_{w}(q) \\
& =E^{w} \cdot\left(\overline{D^{z}} \cdot K_{w \times \bar{z}}(\cdot, \bar{p})\right)(q) \\
& =E^{w} \overline{D^{z}} \cdot K_{w \times \bar{z}}(q, \bar{p}),
\end{aligned}
$$

as desired. This concludes the proof.

COROLIARY 2.6. (Characterization of the Bergman form). The Bergman form $K$ is a unique (2m, 0)-form on the product manifold $M \times \bar{M}$ with the reproducing property, in the sense that $K(\cdot, \bar{p}) \in H(M) \wedge \Lambda_{\bar{p}}^{(m, 0)}(\bar{M})$ for every $p \in M$, and

$$
\alpha_{z}(p)=\left(\alpha, K(\cdot, \bar{p}) / d \bar{z}_{\bar{p}}\right)
$$

for every $\alpha \in H(M)$, and every pair of $p$ and $z$ with $p \in U_{z}$.

Proof. The Bergman form $K$ possesses the reproducing property by Definition 2.3 and Proposition 2.5 (i). The uniqueness of $K$ follows from Aronszajn [1; item (2), p. 343].

PROPOSITION 2.7. Let $\left(\beta_{3}\right)_{j=1}^{N}\left(N \in Z_{+} \cup\{+\infty\}\right)$ be a complete orthonormal system of $H(M)$. If $z$ (resp. $w$ ) is a coordinate around a point $p \in M$ (resp. $q \in M$ ), then the series $\sum_{j=1}^{N}\left(\beta_{j}\right)_{w}(q) \overline{\left(\beta_{j}\right)_{2}(p)}$ converges absolutely to $K_{w \times \bar{z}}(q$, $\bar{p})$, where $K$ is the Bergman form of $M$.

Proof. It follows from (2.6) that the Fourier coefficients $\xi_{\text {j }}$ of $K(\cdot, \bar{p}) / d \bar{z}_{\bar{p}}$ with respect to $\left(\beta_{j}\right)$ are given by $\xi_{j}:=\left(K(\cdot, \bar{p}) / d \bar{z}_{\bar{p}}, \beta_{j}\right)=\overline{\left(\beta_{j}\right)_{z}(p)}$. By the completeness of $\left(\beta_{j}\right)$ we have $\left\|\sum_{j=1}^{n} \xi_{j} \beta_{j}-K(\cdot, \bar{p}) / d \bar{z}_{\tilde{p}}\right\| \rightarrow 0$ as $n \rightarrow N$. Another application of (2.6) gives $\lim _{n \rightarrow N} \sum_{j=1}^{n} \xi_{j}\left(\beta_{j}\right)_{w}(q)=K_{w \times \bar{z}}(q, \bar{p})$, and the result follows.

Remark 2.8. By virtue of Proposition 2.7, the Bergman form introduced in Definition 2.3 coincides, up to a multiplicative constant, with the Bergman kernel form given in Kobayashi [10; p. 269] (see also [13]).

§3. Extremal quantities of the space $H(M)$. We shall first establish a chain rule for the differential operators $\partial_{A}^{z}$ (see $(0.4 .2)$ ). For $n \in Z_{+}$, we denote by $\Pi(n)$ the family of all partitions of the set $\{1, \cdots, n\}(\Pi(0)=\{\phi\})$. Given a multi-index $A=\left(a_{1}, \cdots, a_{n}\right) \in M I(n)$ and a subset $P \subset\{1, \cdots, n\}$, we set $\partial_{A \mid P}^{z}:=$ $\prod_{\imath \in P} \partial_{a_{2}}^{z}$ (when $n=0$, we have $\partial_{\phi i \phi}^{z}=1^{z}$ ).

LEMMA 3.1. Let $z$ and $w$ be coordinates in $M$ with $U_{z} \cap U_{w} \neq \phi$, and let $A \in M I(n)$. Then, for every holomorphic function $f$ on $U_{z} \cap U_{w}$, we have $\partial_{A}^{2} . f$ $=\sum_{\mathscr{Q} \in I(\pi)} f_{A, \mathscr{P}}$, where $f_{A, \mathscr{P}}$ with $\mathscr{P}=\left\{P_{1}, \cdots, P_{u}\right\}$ is the function given by

$$
\sum_{\left(b_{\imath}\right) \in M I(u)}\left(\partial_{A \mid P_{1}}^{2} \cdot w^{b_{1}}\right) \cdots\left(\partial_{A \mid P_{u}}^{2} \cdot w^{b_{u}}\right)\left(\partial_{\left(b_{i}\right)}^{w}, f\right) .
$$

Proof. The proof is carried by induction on $n \in Z_{+}$, using the formula 


$$
\partial_{a_{n+1}}^{z} . f_{A^{\prime}, \mathscr{Q}}=\sum_{\nu=1}^{u} f_{A, \mathscr{Q}(\nu)}+f_{A, \mathscr{Q}} .
$$

Here $A=\left(A^{\prime}, a_{n+1}\right) \in M I(n+1), \mathscr{P}=\left\{P_{1}, \cdots, P_{u}\right\} \in \Pi(n), \mathscr{P}(\nu):=\left\{P_{1}, \cdots, P_{u} \cup\{n+1\}\right.$, $\left.\cdots, P_{u}\right\}$, and $\mathscr{Q}^{\prime}:=\left\{P_{1}, \cdots, P_{u},\{n+1\}\right\}$. The proof is now complete.

DeFinition 3.2. For every $n \in \boldsymbol{Z}_{+}$and $p \in M$, we define a subspace $H_{n}(p)$ of $H(M)$ and a condition $\left(C_{n}\right)_{p}$ as follows:

$$
H_{n}(p):=\left\{\alpha \in H(M) ; \partial_{A}^{2} \cdot \alpha(p)=0\left(A \in \bigcup_{\jmath=0}^{n-1} M I(j)\right)\right\} \quad\left(H_{0}(p)=H(M)\right),
$$

$\left(C_{n}\right)_{p}:\left(\begin{array}{l}\text { For every vector }\left(\xi^{A}\right)_{A \in M I I(n)} \in C^{N}-\{0\}, \text { there exists a form } \alpha \in H_{n}(t) \\ \text { such that } \sum_{A} \xi^{A} \partial_{A}^{z}, \alpha(p) \neq 0 .\end{array}\right.$

Here, $z$ is an arbitrary fixed coordinate around $p$ and $N:=\varphi(n)-\varphi(n-1$ ) (see (0.3.3)). Condition $\left(C_{n}\right)$ stands for the collection of all $\left(C_{n}\right)_{p}(p \in M)$.

By Lemma 3.1, we see that the definitions of $H_{n}(p)$ and $\left(C_{n}\right)_{p}$ do not depend on the choice of the coordinate $z$.

Remark 3.3. Condition $\left(C_{0}\right)$ (resp. $\left(C_{1}\right)$ ) coincides with condition (A. 1) (resp. (A. 2)) of Kobayashi [10].

LEMMA 3.4. Let $K$ be the Bergman form of $M, z$ be a coordinate around a point $p \in M$ and let $n \in Z_{+}$. Set $S(p, z):=\left\{\partial_{A}^{\bar{z}} . K(\cdot, \bar{p}) / d \bar{z}_{\bar{p}} ; A \in \bigcup_{j=u}^{n} M I I(j)\right\} \subset$ $H(M)$. Then:

(i) The space $H_{n+1}(M)$ conncides with $S(p, z)^{\perp}$, the orthogonal subspace of the subset $S(p, z)$ in $H(M)$.

(ii) Conditions $\left(C_{j}\right)_{p}(j=0, \cdots, n)$ hold true af and only if the system $S(p, z)$ is linearly independent in $H(M)$.

Proof. By Proposition 2.5 (i),

$$
\partial_{A}^{z} \cdot \alpha_{z}(p)=\left(\alpha, \overline{\partial_{A}^{\bar{z}}} \cdot K(\cdot, \bar{p}) / d \bar{z}_{\bar{p}}\right), \quad \alpha \in H(M) .
$$

Thus, assertion (i) follows immediately from (3.1). To prove part (ii), suppose that $\left(C_{\jmath}\right)_{p}(j=0, \cdots, n)$ hold true, and let

$$
\sum_{j=0}^{n} \sum_{A \in M I I(\jmath)} \xi^{A} \partial_{A}^{\bar{z}} \cdot K(\cdot, \bar{p}) / d \bar{z}_{\bar{p}}=0
$$

for a vector $\left(\xi^{A}\right)$. It follows from (3.1) that

$$
\sum_{j=0}^{n} \sum_{A \in M I I(j)} \xi^{A} \partial_{A}^{z} \cdot \alpha_{z}(p)=0, \quad \alpha \in H(M) .
$$

Applying formula (3.2) on $\alpha \in H_{n}(p)$ and using assumption $\left(C_{n}\right)_{p}$, we find that $\xi^{A}=0$ for every $A \in M I I(n)$. Similarly and inductively, we conclude that $\xi^{A}=0$ for every $A$. Conversely, suppose that

$S(p, z)$ is linearly independent in $H(M)$,

and let 


$$
\sum_{A \in M I I(j)} \xi^{A} \partial_{A}^{z} \cdot \alpha(p)=0 \quad\left(\alpha \in H_{j}(p)\right),
$$

where $\jmath \in\{0, \cdots, n\}$ and $\xi^{A} \in C$. Substituting (3.1) into formula (3.4), we see that $\sum_{A \in M I Y(j)} \xi^{A} \bar{\partial}_{A}^{z} \cdot K(\cdot, \bar{p}) / d \bar{z}_{\bar{p}} \in H_{j}(p)^{\perp}$. From part (i) with $j$ instead of $n$, assumption (3.3) implies that $\xi^{A}=0$ for every $A$. This concludes the proof.

LEMMA 3.5. Let $X \in T_{p}(M)$ and $\alpha \in H_{n}(p)$. If we express $X=\left(\partial_{v}^{z}\right)_{p}=\left(\partial_{v^{\prime}}^{w}\right)_{p}$ $\left(v, v^{\prime} \in C^{m}\right)$ with respect to coordinates $z$ and $w$ around $p$, then $\left(\partial_{v}^{z}\right)^{n} \cdot \alpha(p)=$ $\left(\partial_{w^{\prime}}^{w}\right)^{n} \cdot \alpha(p)$; therefore, this form at $p$ may be denoted by $X^{n} \cdot \alpha(p)$.

Proof. We first note that

$$
\begin{gathered}
v^{\prime a}=\partial_{v}^{z} \cdot w^{a}(p) \quad(a=1, \cdots, m), \\
\left(\partial_{v}^{z}\right)^{n} \cdot \alpha_{z}(p)=\sum_{\jmath=0}^{n}\left(\begin{array}{c}
n \\
\jmath
\end{array}\right)\left(\partial_{v}^{z}\right)^{n-\jmath} \cdot J_{z}^{w}(p)\left(\partial_{v}^{z}\right)^{\jmath} \cdot \alpha_{w}(p),
\end{gathered}
$$

since $\alpha_{z}=\int_{z}^{w} \alpha_{w}$ (see (0.2.3)). Since $\alpha \in H_{n}(p)$, it follows from Lemma 3.1 as well as (3.5) that

$$
\left(\partial_{v}^{z}\right)^{\jmath} \cdot \alpha_{w}(p)= \begin{cases}0, & \jmath \leqq n-1 \\ \left(\partial_{v^{\prime}}^{w}\right)^{\jmath} \cdot \alpha_{w}(p), & \jmath=n .\end{cases}
$$

Substituting these values into formula (3.6), we obtain

$$
\left(\partial_{v}^{z}\right)^{n} \cdot \alpha_{z}(p)=J_{z}^{w}(p)\left(\partial_{v^{\prime}}^{w}\right)^{n} \cdot \alpha_{w}(p), \quad \text { or } \quad\left(\partial_{v}^{z}\right)^{n} \cdot \alpha(p)=\left(\partial_{v^{r}}^{z}\right)^{n} \cdot \alpha(p),
$$

as desired.

DEFinition 3.6. (Kobayashi $[10 ;$ p. 269]). We define an order relation on the subset $\left\{\omega \wedge \bar{\omega} ; \omega \in \Lambda_{p}^{(m, 0)}(M)\right\} \subset \Lambda_{p}^{(m, m)}(M)$ as follows (see (0.2.2)): We let $\omega \wedge \bar{\omega} \leqq \omega^{\prime} \wedge \bar{\omega}^{\prime}$, for $\omega, \omega^{\prime} \in \Lambda_{p}^{(m, 0)}(M)$, if $\left|\omega_{z}\right| \leqq\left|\omega_{z}^{\prime}\right|$ for some coordinate $z$ around $p$, where $\omega=\omega_{z} d z_{p}, \omega^{\prime}=\omega_{z}^{\prime} d z_{p}\left(\omega_{z}, \omega_{z}^{\prime} \in C\right)$.

Proposition 3.7. For every $X \in T_{p}(M)$ and every $n \in \boldsymbol{Z}_{+}$, the maximum

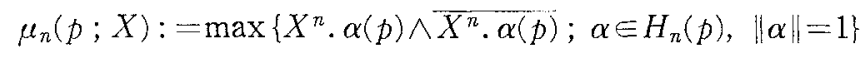

under the order in Definition 3.6 exists and conncides with

$$
\max \left\{|(\beta(z), \alpha)|^{2} ; \alpha \in S(z)^{\perp},\|\alpha\|=1\right\}(d z \wedge \overline{d z})_{p}
$$

for every coordinate $z$ around $p$, where

and

$$
S(z):=\left\{\bar{\partial}_{A}^{z} \cdot K(\cdot, \bar{p}) / d \bar{z}_{\bar{p}} ; A \in \bigcup_{j=0}^{n-1} M I I(j)\right\} \subset H(M)
$$

$$
\beta(z):=\overline{\left(\partial_{v}^{z}\right)^{n}} . K(\cdot, \bar{p}) / d \bar{z}_{\bar{p}} \in H(M), \quad X=\left(\partial_{v}^{z}\right)_{p} .
$$

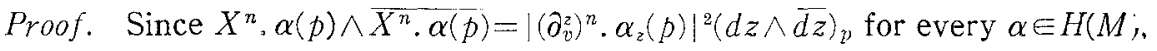


the assertion follows from Proposition 2.5 (i) and Lemma 3.4 (i).

Let $p \in M$. From the definition we deduce the following:

$$
\text { (When } n=0 \text { or } 1, \mu_{n}(p ; X) \neq 0 \text { for every } X \subseteq T_{n}(M)-\{0\}
$$

if and only if $\left(C_{n}\right)_{p}$ holds true;

$$
\begin{aligned}
& \text { When } n \geqq 2, \mu_{n}(p ; X) \neq 0 \text { for every } X \in T_{p}(M)-\{0\} \\
& \text { if }\left(C_{n}\right)_{p} \text { holds true. }
\end{aligned}
$$

To study the $\mu_{n}$ more precisely, we record a lemma which is valid for any pre-Hilbert space $H$. We denote by $G\left(x_{1}, \cdots, x_{n}\right)$ the Gramian of a system $\left(x_{1}, \cdots, x_{n}\right)$ in $H$ (especially, $G(\phi)=1$ ), and denote by $G_{l_{3}}\left(x_{1}, \cdots, x_{n}\right)$ the $(i, j)$ cofactor of the Gram-matrix of $\left(x_{1}, \cdots, x_{n}\right)$ (especially, $G_{11}\left(x_{1}\right)=1$ ).

LEMMA 3.8. Let $\left(x_{1}, \cdots, x_{n}\right)\left(n \in Z_{+}\right)$be a linearly independent system $n$ a pre-Hilbert space $H$, and let $x_{n+1} \in H$. Then

$$
\begin{gathered}
\max \left\{\left|\left(y, x_{n+1}\right)\right|^{2} ; y \in\left\{x_{1}, \cdots, x_{n}\right\}^{\perp},\|y\|=1\right\} \\
=G\left(x_{1}, \cdots, x_{n+1}\right) / G\left(x_{1}, \cdots, x_{n}\right),
\end{gathered}
$$

and the latter coincides with $\left\|y^{(n)}\right\|^{2}$, where

$$
y^{(n)}:=G\left(x_{1}, \cdots, x_{n}\right)^{-1} \sum_{j=1}^{n+1} G_{n+1, j}\left(x_{1}, \cdots, x_{n+1}\right) x_{j} .
$$

Furthermore, when $y^{(n)} \neq 0$, the above maximum is attanned by $y$ if and only if $y=e^{\sqrt{-1} \theta} y^{(n)} /\left\|y^{(n)}\right\|$ for some real $\theta$

DEFINITION 3.9. Let $K$ be the Bergman form of $M$, and let $z$ be a coordinate. Then $\left.K\right|_{U_{z} \times \overline{\theta_{z}}}=K_{z \times \bar{z}} d z \wedge d \bar{z}$. We consider the function $k_{z}$ on $U_{z}$ given by

$$
k_{z}(p):=K_{z: \bar{z}}(p, \bar{p}) \quad\left(p \in U_{z}\right) .
$$

which we call the Bergman function of $M$ relative to $z$.

DEFinition 3.10. Let $\varphi$ and $\Phi$ be as in (0.3.3) and (0.3.4), respectively. For a coordinate $z$ in $M$, we set:

$$
\begin{aligned}
& k_{z, 2 j}:=\partial_{\phi(z)}^{z} \partial_{\phi(j)} \cdot k_{z}, \\
& L_{z}\left(\jmath_{1}, \cdots, \jmath_{n}\right):=\left[k_{z, i j}\right]_{j=\jmath_{1}, \cdots, \cdots, j_{n},}^{2=3,}, \\
& L_{2}\left(\jmath_{1}, \cdots, \jmath_{n}\right)_{s, t}:=\operatorname{det}\left[k_{3,2 j}\right]_{j=j_{1}, \cdots, j_{n}, \imath,}^{2=j_{1}, \cdots, j_{n}, 8} \\
& K_{z, \bar{i}}(p):=\overline{\partial_{\bar{\phi}(i)}} . K(\cdot, \bar{p}) / d \bar{z}_{\bar{p}} \in H(M) \quad\left(p \in U_{z}\right) .
\end{aligned}
$$

It follows from Proposition 2.5 (ii) that $k_{z, i j}=\left(K_{z, j}, K_{z, i}\right)$ on $U_{z}$. This means that the matrix $L_{z}\left(j_{1}, \cdots, j_{n}\right)(p)$ is the transpose of the Gram-matrix of the system $\left(K_{z}, \overline{\gamma_{1}}, \cdots, K_{z}, \overline{\jmath_{n}}\right)$ in $H(M)$ for every $p \in U_{z}$. Combining this with Lemma 3.4 (ii) and Lemma 3.8, we obtain the following two results. 
Proposition 3.11. Let $z$ be a coordinate around $p \in M$, and let $n \in Z_{+}$. Then $L_{z}(1, \cdots, \varphi(n))(p) \in P s(\varphi(n), C)$ (see $\left.(0.1 .1)\right)$, and the following four conditions are mutually equivalent:

(a) Conditions $\left(C_{j}\right)_{p}(j=0, \cdots, n)$ hold true.

(b) The system $\left(K_{z, 1}(p), \cdots, K_{z, \overline{\varphi(n)}}(p)\right)$ in $H(M)$ is linearly independent.

(c) $L_{z}(1, \cdots, \varphi(n))(p) \in P(\varphi(n), C)$.

(d) $\operatorname{det} L_{z}(1, \cdots, \varphi(n))(p)>0$.

THEOREM 3.12. Let $z$ be a coordinate in $M$ and let $f_{n, z}$ be the function on $U_{z} \times C^{m}$, defined by

$$
\mu_{n}\left(p ;\left(\partial_{v}^{z}\right)_{p}\right)=f_{n, z}(p, v)(d z \wedge d \overline{d z})_{p}, \quad(p, v) \in U_{z} \times C^{m} .
$$

Then, for every $p \in U_{z}$ and any maximal linearly independent subset $\left\{K_{z}, \bar{J}_{1}(p), \cdots\right.$, $\left.K_{z, \bar{\jmath}}(p)\right\}$ of $\left\{K_{z, \mathrm{i}}(p), \cdots K_{z}, \overline{\varphi(n-1)}(p)\right\}$,

$$
\begin{aligned}
& f_{n, z}(p, v)=\operatorname{det} L_{2}\left(\jmath_{1}, \cdots, \jmath_{l}\right)(p)^{-1} \\
& \quad \times \sum_{\varphi(n-1)<s_{2} t \leq \varphi(n)} C_{\Phi(s)} C_{\Phi(t)} v^{\Phi(s)} \bar{v}^{\Phi(t)} L_{z}\left(\jmath_{1}, \cdots, \jmath_{l}\right)_{s, t}(p) .
\end{aligned}
$$

Here, $C_{A}=n ! / n_{1} ! \cdots n_{m} !, v^{A}=v^{a_{1}} \cdots v^{a_{n}}$ for $A=\left(a_{1}, \cdots, a_{n}\right)$ and $v=\left(v^{1}, \cdots, v^{m}\right)$, where $n_{\nu}$ is the cardinarity of the set $\left\{0 ; a_{j}=\nu\right\}$.

Corollary 3.13. (Kobayashi [10; Theorem 2.2]). For $p \in M$,

$$
K(p, \bar{p})=\max \{\alpha(p) \wedge \overline{\alpha(p)} ; \alpha \in H(M),\|\alpha\|=1\} .
$$

If $K(p, \bar{p}) \neq 0$, the above maximum is attained by $\alpha$ if and only if $\alpha=$ $e^{\sqrt{ }-1 \theta} k_{z}(p)^{-1} K(\cdot, \bar{p}) / d \bar{z}_{\bar{p}}$ for some real $\theta$.

Proof. The first assertion follows from Theorem 3.12 with $n=0$, and the latter from Lemma 3.8 with $n=0$.

$\S 4$. The biholomorphic invariant $\mu_{0, n}$. In this section we suppose that $M$ satisfies condition $\left(C_{0}\right)$, i. e. $M$ satisfies condition (A. 1) of Kobayashi [10] (see Remark 3.3). For every $n \in Z_{+}$and every $X \in T_{p}(M)$, the $(n, n)$-form

$$
\mu_{n}(p ; X)=\max \left\{X^{n} . \alpha(p) \wedge X^{n} \cdot \alpha(\bar{p}) ; \alpha \in H_{n}(p),\|\alpha\|=1\right\}
$$

at $p$ has been defined in Proposition 3.7. When $n=0$, by Corollary 3.13 together with $(3.7)_{1}$, we have

$$
\mu_{0}(p ; X)=k_{z}(p)(d z \wedge \bar{d} \bar{z})_{p}, \quad k_{z}(p)>0 .
$$

Definition 4.1. For every $n \in \boldsymbol{N}$, we let $\mu_{0, n}:=\mu_{n} / \mu_{0}$. Thus it follows that $\mu_{0, n}$ is a well-defined $[0,+\infty)$-valued function on the tangent bundle $T(M)$, for which, by (4.1), it possesses the property that for every $X \in T_{p}(M)$ and every 
$\xi \in C, \mu_{0, n}(p ; \xi X)=|\xi|^{2 n} \mu_{0, n}(p ; X)$.

THEOREM 4.2. The function $\mu_{0, n}$ on $T(M)$ is a biholomorphic invariant, i.e. $\mu_{0, n}(p ; X)=\mu_{0, n}\left(f(p) ; f_{*} X\right)((p ; X) \in T(M))$ for every biholomorphic mapping $f$ from $M$ onto the complex manifold $f(M)$.

Proof. Let $M^{\prime}:=f(M)$ and let $q:=f(p)$. The mapping $f$ induces an isometry $f^{*}$ of the Hilbert space $H\left(M^{\prime}\right)$ onto $H(M)$ so that $f^{*} H_{n}(q)=H_{n}(p)$. Let $\left(w, U_{w}\right)$ be a chart of $M^{\prime}$ around $q$. Then, the function $z:=\left.w \circ f\right|_{U_{z}}$ with $U_{z}$ : $=f^{-1}\left(U_{w}\right)$ is a coordinate around $p$ such that

$$
z^{a}=w^{a} \circ \quad \text { on } U_{z} \quad(a=1, \cdots, m) .
$$

Let $X=\left(\partial_{v}^{z}\right)_{p} \in T_{p}(M)$. Thus, by (4.2), $f_{*} X=\left(\partial_{v}^{w}\right)_{q}$. Furthermore, by induction on $n$ and by virtue of (4.2), we obtain, for every $\alpha \in H_{n}(q)$,

$$
\left(\partial_{v}^{z}\right)^{n} \cdot\left(f^{*} \alpha\right)_{z}=\left(\partial_{v}^{z}\right)^{n} \cdot\left(\alpha_{w} \circ f\right)=\left(\left(\partial_{v}^{w}\right)^{n} \cdot \alpha_{w}\right) \circ f \quad \text { on } U_{z} .
$$

Evaluating the above formula at the point $p$, we obtain that $\left(\partial_{0}^{z}\right)^{n} \cdot\left(f^{*} \alpha\right)_{z}(p)$ $=\left(\partial_{v}^{w}\right)^{n} \cdot \alpha_{w}(q)$ for every $\alpha \in H_{n}(q)$. It follows from (4.1) that

$$
\mu_{n}(p ; X) /(d z \wedge \overline{d z})_{p}=\mu_{n}\left(q ; f_{*} X\right) /(d w \wedge \overline{d w})_{q} .
$$

The desired assertion follows now from Definition 4.1.

Remark 4.3. Let $C(p ; X)$ be the Carathéodory metric on $M$. Suppose that $\left(C_{0}\right)_{p}$ holds and $C(p ; X)>0$ for some $(p ; X) \in T(M)$. Then the same argument as in the proof in $\left[6 ;\right.$ Theorem 1] implies that $C(p ; X)^{2 n}<(n !)^{-2} \mu_{0, n}(p ; X)$ for every $n \in N$.

Now, making use of Theorem 3.13 , we have

$$
\mu_{0,1}(p ; X)=\partial_{z}^{z} \overline{\partial_{v}^{z}} \cdot \log k_{z}(p), \quad X=\left(\partial_{v}^{z}\right)_{p} \in T_{p}(M) .
$$

With the aid of the above formula, one can extend $\mu_{0,1}$ to a unique hermitian pseudo-metric $g$ on $M$ such that $g(X, \bar{X})=\mu_{0,1}(p ; X), X \in T_{p}(M)$. This pseudometric is given by

$$
\left.g\right|_{b_{z}}=2 \sum_{a, b} \partial_{a}^{z} \bar{\partial}_{b}^{z} \cdot \log k_{z} d z^{a} \cdot d \bar{z}^{b},
$$

and is called the Bergman pseudo-metric on $M$. We note that the Bergman pseudo-metric $g$ becomes an ordinary metric if and only if $M$ satisfies condition $\left(C_{1}\right)$ (see $\left.(3.7)_{1}\right)$, i.e. $M$ satisfies condition (A.2) of Kobayashi [10] (see Remark $3.3)$.

Assume now that $M$ satisfies condition $\left(C_{1}\right)$. It follows from Theorem 3.12 that

$$
\mu_{0,2}\left(p ;\left(\partial_{v}^{z}\right\rangle_{p}\right)=k_{z}(p)^{-1} P_{z}(p)^{-1} Q_{z}(p, v),
$$

where 
and

$$
P_{z}:=\operatorname{det} L_{z}(1, \cdots, \varphi(1))
$$

$$
Q_{z}(\cdot, \nu):=\sum_{\varphi(1)<s, t \leqslant \psi_{(2)}} C_{\phi(s)} C_{\phi(t)} v^{\phi(s)} \tilde{v}^{\phi(t)} L_{z}(1, \cdots, \varphi(1))_{s, t} .
$$

The following theorem was stated in Fuks [8; p. 525]. For the sake of completeness we give another proof which may have its own interest.

THEOREM 4.4. Suppose $M$ satisfies conditions $\left(C_{0}\right)$ and $\left(C_{1}\right)$. Let $\operatorname{Sec}(p ; \cdot)$ be the curvature quartic form, at $p \in M$, of the Bergman metric $g$ on $M$ (see Definitoon 1.1). Then,

$$
\mu_{02}(p ; X)=2 g(X, \bar{X})^{2}-\operatorname{Sec}(p ; X), \quad X \in T_{p}(M) .
$$

Proof. Set $g_{z, a \bar{b}}:=\partial_{\alpha}^{z} \dot{\partial}_{\bar{b}}^{\bar{z}} \cdot \log k_{z}, \quad G_{z}:=\left(g_{z, a \tilde{b}}\right), \quad\left(g_{z}^{\bar{b} a}\right):=G_{z}^{-1}$. We compute $\mu_{0,2}\left(p ;\left(\partial_{v}^{z}\right)_{p}\right)$ with the aid of formula (4.3). We first note that

$$
\begin{gathered}
P_{z}=k_{z}^{m+3} \operatorname{det} G_{z}, \\
Q_{z}(\cdot, v)=k_{z}^{m+1} \operatorname{det}\left[\begin{array}{ll}
G_{z} & x_{z, v}^{*} \\
x_{z, v} & \sigma_{z, v}
\end{array}\right],
\end{gathered}
$$

where $x_{z, v}$ and $\sigma_{z, v}$ are $C^{m}$-valued and $C$-valued functions on $U_{z}$, respectively, given by

It follows that

$$
\begin{gathered}
x_{z, v}:=\left(\partial_{b}^{z} \cdot\left(\left(\partial_{v}^{z}\right)^{2} \cdot k_{z} / k_{z}\right)\right)_{b}, \\
\sigma_{z, v}:=\left(k_{z}\left(\partial_{v}^{z}\right)^{2}\left(\partial_{v}^{2}\right)^{2} \cdot k_{z}-\left|\left(\partial_{v}^{z}\right)^{2} \cdot k_{z}\right|^{2}\right) / k_{z}^{2} .
\end{gathered}
$$

$$
\mu_{0,2}\left(p ;\left(\partial_{v}^{z}\right)_{p}\right)=\sigma_{z} v(p)-x_{z, v}(p) G_{z}(p)^{-1} x_{z, v}(p)^{*} .
$$

The desired formula is now obtained from Definition 1.1 (see also [10; p. 275]), and the proof is complete.

Corollary 4.5. (Fuks [8; Theorem 1], Kobayashi [10; Theorem 4.4]). Suppose $M$ satisfies conditions $\left(C_{0}\right)$ and $\left(C_{1}\right)$. Then the holomorphic sectional curvature of the Bergman metrac on $M$ is at most 2. Let $p \in M$ be fixed. The holomorphic sectional curvature is less than 2 for every direction at $p$ if condition $\left(C_{2}\right)_{p}$ holds.

Remark 4.6. Concerning the last corollary, the following facts are shown in [2] by means of examples:

(i) There exists a simply connected domain $M$ in $C^{2}$ such that conditions $\left(C_{0}\right)$ and $\left(C_{1}\right)$ hold true, and such that the holomorphic sectional curvature of the Bergman metric on $M$ is identically 2.

(ii) For every real number $\xi$ with $\xi<2$, there exists a pseudo-convex bounded Reinhardt domain $M$ in $C^{2}$ such that the holomorphic sectional curvature of the Bergman metric on $M$ is greater than $\xi$ for some direction. 
$\S 5$. Hessian quartic form of the Bergman metric. We first recall the $n$-th order Bergman metric introduced in [6]. Let a coordinate $z$ in $M$ be fixed. For $n \in \boldsymbol{Z}_{+}$and $(p, v) \in U_{2} \times \boldsymbol{C}^{m}$, we set

and

$$
H_{n}^{2}(p, v):=\left\{\alpha \in H(M) ;\left(\partial_{v}^{2}\right)^{3} \cdot \alpha(p)=0 \quad(j=1, \cdots, n-1)\right\}
$$

$$
\lambda_{n}^{z}\left(p ;\left(\partial_{v}^{z}\right)_{p}\right):=\max \left\{\left(\partial_{v}^{z}\right)^{n} \cdot \alpha(p) \wedge\left(\overline{\left.\partial_{v}^{z}\right)^{n} \cdot \alpha(p)} ; \alpha \in H_{n}^{2}(p, v),\|\alpha\|=1\right\}\right.
$$

(see Definition 3.6). Referring to Definition 3.2, we have

$$
H_{n}^{z}(p, v) \begin{cases}=H_{n}(p), & n=0,1 \\ \supset H_{n}(p), & n \geqq 2 .\end{cases}
$$

In particular,

$$
\left\{\begin{array}{l}
\lambda_{0}^{z}(p ; \cdot)=\mu_{0}(p ; \cdot)=k_{z}(p)(d z \wedge d z)_{p} \\
\lambda_{1}^{z}(p ; \cdot)=\mu_{1}(p ; \cdot)
\end{array}\right.
$$

on $T_{p}(M)$. When $M$ satisfies condition $\left(C_{0}\right)$, we may consider the $[0,+\infty)$-valued function $\lambda_{0, n}^{z}$ on $\bigcup_{p \in U_{z}} T_{p}(M)$ for every $n \in N$, given by $\lambda_{0, n}^{z}=\lambda_{n}^{z} / \lambda_{0}^{z}$. The function $\lambda_{0, n}^{2}$ is called in [6] the $n$-th order Bergman metric of $M$. It follows from (5.1) and (5.2) that

$$
\lambda_{0,1}^{z}=\mu_{0,1}, \quad \lambda_{0, n}^{z} \geqq \mu_{0, n} \quad(n \geqq 2) .
$$

Given a vector $v \in C^{m}$, consider the functions $R_{n}(n=-1,0,1, \cdots)$ on $U_{z}$ given by

$$
R_{n}:=\operatorname{det}\left[\left(\partial_{v}^{z}\right)^{2}\left(\overline{\left.\partial_{v}^{2}\right)^{3}}, k_{z}\right]_{\jmath=0, \cdots, \cdots, n}^{\imath=0,}\right.
$$

the Wronskian of functions $\left(\overline{\left.\partial_{v}^{z}\right)^{2}} \cdot k_{z}(\jmath=0,1, \cdots, n)\right.$ with respect to $\partial_{v}^{z}$ (especially, $R_{-1}=1$ ).

We now recall the Jacobi's formula concerning determinants.

Lemma 5.1. Let $A=\left(\xi_{\imath \jmath}\right) \in M(n, C)$, and let $A_{\imath \jmath}$ be its $(i, j)$-cofactor. Then $\operatorname{det} A \operatorname{det}\left(\xi_{2,}\right)_{j=1, \cdots ; n-2}^{2=1, \cdots, n-2}=A_{n n} A_{n-1, n-1}-A_{n, n-1} A_{n-1, n}$.

This lemma leads to the following recursive formula for the Wronskians $R_{n}$ in (5.4).

Lemma 5.2. Let $z$ be a coordinate in $M$, and let $v \in C^{m}$. Then, for every $n \in N$,

on $U_{z}$.

$$
R_{n} R_{n-2}=R_{n-1} \partial_{v}^{2} \bar{\partial}_{v}^{z} \cdot R_{n-1}-\left|\partial_{v}^{z} \cdot R_{n-1}\right|^{2}
$$

Proof. Let $\left(R_{n}\right)_{i}$ be the $(\imath, j)$-cofactor of the $H(n+1, C)$-valued function 
$\left[\left(\partial_{0}^{2}\right)^{2}\left(\overline{\partial_{0}^{2}}\right)^{n}, k_{z}\right]_{\jmath=0, \cdots, n}^{2=0, \cdots, n}$ It follows from Lemma 5.1 , since $R_{n}$ is hermitian, that

$$
R_{n} R_{n-2}=\left(R_{n}\right)_{n n}\left(R_{n}\right)_{n+1, n+1}-\left|\left(R_{n}\right)_{n, n+1}\right|^{2} \text {. }
$$

Moreover, from the derivation properties of the Wronskians we also have $\left(R_{n}\right)_{n n}=R_{n-1},\left(R_{n}\right)_{n, n+1}=-\hat{\partial}_{v}^{z} . R_{n-1}$, and $\left(R_{n}\right)_{n+1, n+1}=\partial_{v}^{z} \overline{\partial_{v}^{z}} \cdot R_{n-1}$. The proof is now complete.

From Lemma 3.8 together with (5.2) it follows that

$$
\lambda_{0, n}^{z}\left(p ;\left(\partial_{v}^{z}\right)_{p}\right)=k_{z}(p)^{-1} R_{n-1}(p)^{-1} R_{n}(p),
$$

provided that $R_{n-1}(p) \neq 0$ (cf. $[6 ;$ p. 51$]$ ).

THEOREM 5.3. Assume, in addition to the assumptions of Lemma 5.2, that $M$ satisfies condition $\left(C_{j}\right)(\jmath=0, \cdots, n-1)$. Set

Tho?!

$$
\lambda_{0, j}(p):=\lambda_{0,3}^{z}\left(p ;\left(\partial_{v}^{z}\right)_{p}\right), \quad p \in L_{z} \quad(=1, \cdots, n) .
$$

$$
\lambda_{1, n}=\lambda_{0, n-1}\left(n \lambda_{1,1}+\sum_{j=1}^{n-1} \hat{\alpha}_{v}^{z} \ddot{\partial}_{v}^{z} \cdot \log \lambda_{0, j}\right)
$$

on $U_{z}^{r}$. where $\lambda_{0,0}=1$.

Proof. By assumption and Lemma 5.2 we have

$$
R_{n} R_{n-2}=\left(R_{n-1}\right)^{2} \partial_{z}^{z} \bar{\partial}_{v}^{z} \cdot \log R_{i n-1} .
$$

It follows from (5.5) that

and that

$$
\lambda_{0, n}=\lambda_{0, n-1} \hat{\alpha}_{v}^{z} \hat{\partial}_{v}^{z} \cdot \log R_{n-1}
$$

$$
R_{n-1}=\left(k_{z}\right)^{n} \lambda_{0,1} \cdots \lambda_{v, n-1} \text {. }
$$

The desired result now follows by observing that $\lambda_{0,1}=\partial_{v}^{z} \partial_{v}^{z} \cdot \log k_{z}$.

As a consequence of this theorem we find an intimate relationship between the quantity $\lambda_{0,2}^{z}$ and the Hessian quartic form of the Bergman metric.

Corollary 5.4. Suppose that $M$ satisfies conditions $\left(C_{0}\right)$ and $\left(C_{1}\right)$. Let $z$ be a coordinate in $M$, and let $\operatorname{Hess}^{z}(\cdot ; \cdot)$ be the Hessian quartic form of the Bergman metric $g$ on $M$, relative to $z$ (see Definition 1.3). Then, for $(p, v) \in U_{z} \times \boldsymbol{C}^{m}$,

$$
\lambda_{0,2}^{z}\left(p ;\left(\partial_{v}^{z}\right)_{p}\right)=2 g\left(\left(\hat{\partial}_{v}^{z}\right)_{p},\left(\partial_{v}^{z}\right)_{p}\right)^{2}-\operatorname{Hess}^{z}\left(p ;\left(\partial_{v}^{z}\right)_{p}\right) .
$$

Combining Theorem 4.3 with Corollary 5.4 , we obtain, for $(p, v) \in U_{z} \times C^{m}$,

$$
\operatorname{Sec}\left(p ;\left(\partial_{v}^{z}\right)_{p}\right)-\operatorname{Hess}^{z}\left(p ;\left(\partial_{v}^{z}\right\rangle_{p}\right)=\lambda_{0,2}^{z}\left(p ;\left(\partial_{v}^{z}\right)_{p}\right)-\mu_{0,2}\left(p ;\left(\partial_{v}^{z}\right)_{p}\right) \geqq 0 .
$$

The latter inequality follows from Proposition 1.5 or (5.3).

Proposition 5.5. Suppose that $M$ satisfies conditions $\left(C_{0}\right)$ and $\left(C_{1}\right)$. Let $z$ be a coordinate in $M$ and let $\operatorname{Sec}(\cdot ; \cdot)\left(\right.$ resp. $\left.\operatorname{Hess}^{2}(\cdot ; \cdot)\right)$ be the curvature quartic 
form (resp. Hessian quartic form relalive to $z$ ) of the Bergman metric $g$ on $M$. Let $(p, v) \in U_{z} \times C^{m}$ be fixed. Then, the left hand side of (5.6) vanishes if and only if

$$
W_{v}^{z}\left(k_{z}, \overline{\partial_{a}^{z}} \cdot k_{z}, \partial_{\bar{b}}^{\bar{z}}, k_{z}\right)(p)=0 \quad(a, b \in\{1, \cdots, m\}),
$$

where $W_{v}^{z}\left(f_{0}, \cdots, f_{n}\right)$ is the Wronskian of functions $f_{0}, \cdots, f_{n}$ on $U_{2}$ with respect to $\partial_{v}^{z}$. Condition (5.7) is equivalent to

$$
\operatorname{rank}\left[\begin{array}{l}
\left(k_{z}, \partial_{v}^{z} \cdot k_{z},\left(\partial_{v}^{z}\right)^{2} \cdot k_{z}\right) \\
\left(\overline{\partial_{a}^{z}} \cdot k_{z}, \overline{\partial_{a}^{z}} \partial_{v}^{z} \cdot k_{z}, \overline{\partial_{a}^{z}}\left(\partial_{v}^{z}\right)^{2} \cdot k_{z}\right)_{a=1, \ldots, m}
\end{array}\right](p) \leqq 2 .
$$

Proof. We suppress the dependence on $z$. Set $g_{a \bar{b}}:=\partial_{a} \overline{\partial_{b}} \cdot \log k$ and $G:=$ $\left(g_{a \bar{b}}\right)$. From Proposition 1.5 it follows that equality in (5.6) holds if and only if $v \partial_{v} . G(p)=\xi G(p)$ for some scalar $\xi \in C$. The latter is equivalent to

$$
W_{v}\left(\bar{\partial}_{a} \partial_{v} \cdot \log k, \bar{\partial}_{b} \partial_{v} \cdot \log k\right)(p)=0 \quad(a, b \in\{1, \cdots, m\}) .
$$

But, using Lemma 5.1 with $n=3$ and standard properties of Wronskians, we arrive at the following identity:

$$
W_{v}\left(k, \overline{\partial_{a}} \cdot k, \overline{\partial_{t}} \cdot k\right)=k^{3} W_{v}\left(\overline{\partial_{a}} \partial_{v} \cdot \log k, \overline{\partial_{b}} \partial_{v} \cdot \log k\right) .
$$

It follows that condition (5.9) is equivalent to (5.7).

It remains to show the equivalence of conditions (5.7) and (5.8). Clearly, (5.8) implies (5.7). Assume now that (5.7) holds and $v \neq 0$. Consider the vectors $x:=\left(k, \partial_{v} . k,\left(\partial_{v}\right)^{2} \cdot k\right)(p), y:=\overline{\partial_{v}} \cdot\left(k, \partial_{v} . k,\left(\partial_{v}\right)^{2} . k\right)(p), y_{a}:=\overline{\partial_{a}} \cdot\left(k, \partial_{v} . k,\left(\partial_{v}\right)^{2} \cdot k\right)(p)$ $(a=1, \cdots, m)$ in $C^{3}$. Because of condition $\left(C_{1}\right)_{p}$ which guarantees that $W_{v}\left(k, \bar{\partial}_{v}, k\right)(p) \neq 0$, the set $\{x, y\}$ is linearly independent. It follows, since $y=$ $\Sigma v^{a} y_{a}$, that there exists an $a_{0} \in\{1, \cdots, m\}$ such that $\left\{x, y_{a_{0}}\right\}$ is linearly independent. Therefore, (5.7) implies that every $y_{a}$ is spanned by $x$ and $y_{a_{0}}$, and hence condition (5.8) holds. The proof is now complete.

We note that condition (5.7) holds true trivially when $m=1$.

EXAMPLE 5.6. Suppose that $M=\left\{\left(\xi^{1}, \xi^{2}\right) \in C^{2} ;\left|\xi^{1}\right|^{2}+\left|\xi^{2}\right|^{2 / s}<1\right\}$ for some positive real number $s$, and that the coordinate $z$ is the inclusion mapping of $M$ into $C^{2}$. The Bergman function $k=k_{z}$ of $M$ is given by

$$
k\left(\xi^{1}, \xi^{2}\right)=c \frac{\left(1-\left|\xi^{1}\right|^{2}\right)^{s}-r\left|\xi^{2}\right|^{2}}{\left(\left(1-\left|\xi^{1}\right|^{2}\right)^{s}-\left|\xi^{2}\right|^{2}\right)^{3}\left(1-\left|\xi^{1}\right|^{2}\right)^{2-s}},
$$

where $c:=(1+s) / \pi^{2}=\operatorname{vol}(M)^{-1}$ and

$$
r=r(s):=(1-s) /(1+s) \quad(-1<r<1)
$$

(cf. Bergman $\left[4 ;\right.$ p. 21]). Assume that the point $p$ under consideration is $\left(0, \xi^{2}\right)$ with $\left|\xi^{2}\right|<1$. As in [3] (not Definition 3.10), we use the convenient variable 


$$
t:=\frac{1-\left|\xi^{2}\right|^{2}}{1-r\left|\xi^{2}\right|^{2}} \quad(0<t \leqq 1), \quad \text { or } \quad\left|\xi^{2}\right|^{2}=\frac{1-t}{1-r t},
$$

and the notation $k_{a}:=\partial_{a}^{z}, k, k_{a \bar{b}}:=\partial_{a}^{z} \partial_{b}^{z}, k$, etc. Then, we have

$$
\left\{\begin{array}{l}
k_{1} / k=0, \quad k_{2} / k=x_{1} \xi^{2} \\
k_{11} / k=k_{12} / k=0, \quad k_{22} / k=x_{2}\left(\bar{\xi}^{2}\right)^{2} \\
k_{11} / k=x_{3}, \quad k_{1 \overline{2}} / k=0, \quad k_{2 \overline{2}} / k=x_{4} \\
k_{1 \overline{1}} / k=0, \quad k_{1 \overline{1}} / k=x_{5} \xi^{2}, \quad k_{2 \overline{2}} / k=x_{6} \xi^{2}
\end{array}\right.
$$

and their corresponding conjugated formulas, where

$$
\left\{\begin{array}{l}
x_{1}:=(1-r t)(3-r t) /(1-r) t \\
x_{2}:=6(1-r t)^{2}(2-r t) /(1-r)^{2} t^{2} \\
x_{3}:=\left(3+r t^{2}\right) /(1+r) t \\
x_{4}:=(1-r t)\left(12-9(1+r) t+(5+r) r t^{2}\right) /(1-r)^{2} t^{2} \\
x_{5}:=2(1-r t)\left(6-3 r t+r t^{2}\right) /(1+r)(1-r) t^{2} \\
x_{6}:=12(1-r t)^{2}\left(5-(3+5 r) t+(2+r) r t^{2}\right) /(1-r)^{3} t^{3} .
\end{array}\right.
$$

Using (5.12), we find that condition (5.7) is equivalent to

$$
\left|\begin{array}{ccc}
1 & x_{1} \xi^{2} \bar{v}^{2} & x_{2}\left(\xi^{2}\right)^{2}\left(\bar{v}^{2}\right)^{2} \\
0 & x_{3} \bar{v}^{1} & 2 x_{5} \xi^{2} \bar{v}^{1} \bar{v}^{2} \\
x_{1} \bar{\xi}^{2} & x_{4} \bar{v}^{2} & x_{6} \xi^{2}\left(\bar{v}^{2}\right)^{2}
\end{array}\right|=0
$$

If $v^{1} v^{2} \xi^{2}=0$, condition (5.13) holds true trivially. Suppose that $v^{1} v^{2} \xi^{2} \neq 0$. Then (5.13) is equivalent to

$$
\left|\begin{array}{ccc}
\left|\xi^{2}\right|^{-2} & x_{1} & x_{2} \\
0 & x_{3} & 2 x_{5} \\
x_{1} & x_{4} & x_{6}
\end{array}\right|=0
$$

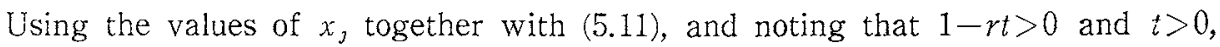
we find that $(5.14)$ is equivalent to

$$
r\left\{9+9(1-r) t-18 r t^{2}-(1-9 r) r t^{3}+r^{2} t^{4}\right\}=0 .
$$

Making use of Sturm's method, we can see that the factor in the brace of (5.15) is positive for every $(r, t) \in(-1,1] \times(0,1]$ (for Sturm's method, cf., e. g., Isaacson and Keller [9; pp. 126-129]); therefore, (5.15) holds if and only if $r=0$, or by (5.10), if and only if $s=1$. Note that the domain $M$ with $s=1$ is the unit ball in $C^{2}$. 
Summing up the above arguments, we obtain the following assertion.

Proposition 5.7. Suppose that $M$ and $z$ are as in Example 5.6 with $s \neq 1$. Let Sec and $\mathrm{Hess}^{z}$ be as in Propostion 5.5, and let $X=\left(\partial_{v}^{z}\right\rangle_{p}$ with $v=\left\langle v^{1}, v^{2}\right) \in C^{2}$ and $p=\left(0, \xi^{2}\right) \in M$. Then, $\operatorname{Sec}(p ; X)-\operatorname{Hess}^{z}(p ; X)=\lambda_{0,2}^{z}(p ; X)-\mu_{0,2}(p ; X)$ is positive if and only if $v^{1} v^{2} \xi^{2} \neq 0$.

It was shown in [6] (see also [5]) that the quantity $\lambda_{\theta_{2}}^{2}$ possesses a certain biholomorphic invariance. This invariance, however, is not an invariance in the ordinary sense and it does not guarantee that for $n \geq 2, \lambda_{0, n}^{2}$ can be regarded as a global function on the tangent bundle $T(M)$ of $M$. In fact, as the following corollary of Proposition 5.7 shows, $\lambda_{0,2}^{z}$ does depend, in general, on the coordinate $z$.

Corollary 5.8. Let $M, z$, Hess ${ }^{2}$ be as in Proposition 5.5 with $m=\operatorname{dim} M \geqq 2$. The quantities $\lambda_{0,2}^{z}$ and $\mathrm{Hess}^{2}$, in general, depend on $z$, i.e. they cannot be considered as global functions on the tangent bundle $T(M)$.

Proof. It is sufficient to find a manifold $M$ that satisfies $\left(C_{0}\right)$ and $\left(C_{1}\right)$, and in which there exist two coordinates $z$ and $w$ with $U_{z} \cap U_{w} \neq \phi$ such that $\lambda_{i, 2}^{z}(p ; X) \neq \lambda_{0,2}^{w}(p ; X)$ for some $p \in U_{z} \cap U_{w}$ and $X=\left(\partial_{v}^{2}\right)_{p}=\left(\partial_{v^{\prime}}^{w}\right)_{p} \in T_{p}(M)$.

For this, we take as $M$ the domain considered in Example 5.6, and as $z$ the inclusion mapping of $M$ into $C^{2}$. We also take $p=\left(0, \xi^{2}\right) \in M$ and $v=\left(v^{1}, v^{2}\right) \in C^{2}$ so that $v^{1} v^{2} \xi^{2} \neq 0$. Lemma 1.6 guarantees the existence of a coordinate $w$ around $p$, for which $\operatorname{Hess}^{w}\left(p ;\left(\partial_{v^{\prime}}^{w}\right)_{p}\right)=\operatorname{Sec}\left(p ;\left(\partial_{v^{\prime}}^{w}\right)_{p}\right)$ with $\left(\partial_{w^{\prime}}^{w}\right)_{p}=\left(\partial_{v}^{z}\right)_{p}$. Then, by $(5.6)$ and Proposition 5.7 we have

as clesired.

$$
\begin{aligned}
& \operatorname{Hess}^{2}\left(p ;\left(\partial_{v}^{z}\right)_{p}\right)<\operatorname{Hess}^{w}\left(p ;\left(\partial_{v^{\prime}}^{w i}\right)_{y}\right), \\
& \lambda_{l, \varepsilon}^{z}\left(p ;\left(\partial_{v}^{z}\right)_{\eta}\right)>\lambda_{0,2}^{w}\left(p ;\left(\partial_{v^{\prime}}^{w}\right)_{\eta^{\prime}}\right),
\end{aligned}
$$

\section{REFERENCES}

[1] N. Aronszajn, Theory of reproducing kernels, Trans. Amer. Math. Soc, 68 (1950), 337-404.

[2] K. AzUKAwA, Square-integrable holomorphic functions on a circular domam in $C^{\text {it }}$ (to appear).

3 K. AzUKawa and M. Suzcki, The Bergman metric on a Thullen domain, Nagoya Math. J., 89 (1983), 1-11.

[4] S. Bergman, Sur les Fonctions Orthagonales de Pluszeurs Varzables Compleses avec les Applications à la Théorie des Fonctzons Analytıques, Mémor Scı. Math., 106, Gauthier-Villars, Paris, 1947.

[5. J. Burbea, The Carathéodory metric and its majorant metrics, Canad. J. Math., 29 (1977), 771-780.

[6] J. Burbea, Inequalities between intrinsic metrics, Proc. Amer Math. Soc, 67 (1977), 50-54.

4.] J. Burbes, On the Hessian of the Carathéociory retric, Rocky Mountan J. 
Math., 8 (1978), 555-559.

[8] B.A. Fuks, Ricc1 curvature of a Bergman metric invariant under biholomorphic mappings, Soviet Math. Dokl., 7 (1966), 525-529.

[9] E. IsaAcson And H.B. Keller, Analysis on Numerical Methods, John Wiley, New York, 1966.

[10] S. Kobayashi, Geometry of bounded domains, Trans. Amer. Math. Soc., 92 (1952), 267-290.

[11] S. KoBAYASHI, Hyperbolic Manifolds and Holomorphic Mappings, Marcel Dekker, New York, 1970.

[12] S. Kobayashi And K. Nomizu, Foundations of Differential Geometry (vol. 2), Interscience, New York, 1969.

[13] A. Lichnerowicz, Variétés complexes et tenseur de Bergmann, Ann. Inst. Fourier (Grenoble), 15 (1965), 345-408.

[14] H. WU, A remark on holomorphic sectional curvature, Indiana Univ. Math. J., 22 (1973), 1103-1108.

Department of Mathematics

TOYAMa UnIVERSTTY

TOYAMA, 930

JAPAN
Department of Mathematics

University of PitTsburgit

Pittsburgh, Pennsylvania 15260

U.S.A. 